$\sqrt{3}$

J. Bio-Sci. 27: 23-30, 2019

ISSN 1023-8654

http://www.banglajol.info/index.php/JBS/index

DOI: https://doi.org/10.3329/jbs.v27i0.44668

\title{
EFFECT OF MEDICINAL PLANT EXTRACTS ON SEED GERMINATION AND SEED ASSOCIATED MYCOFLORA OF WHEAT (TRITICUM AESTIVUM L.)
}

\author{
MF Mahal ${ }^{*}$ and MS Akter \\ Department of Botany, University of Rajshahi, Rajshahi-6205, Bangladesh
}

\begin{abstract}
The experiment was carried to evaluate the efficacy of five different plant extracts i.e. Adhatida vasica, Tagetes patula, Vinca rosea, Lowsonia alba and Andrographis paniculuta on seed germination, vigor index and seed borne and seed associated mycoflora of wheat varieties viz. Sourav and Prodip in in vitro condition. Seed health test of the collected wheat seed revealed that the seed was associated with six different fungi viz. Bipolaris sorokiniana, Fusarium sp., Aspergillus spp., Trichoderma viride, Penicillium sp. and Rhizopus sp. Wheat seeds were treated by dipping separately into different plant extracts of 1:1, 1:2 and 1:3 dilutions. Among the plant extracts, Lowsonia alba leaf extract was observed most effective followed by $A$. vasica, $T$. patula, $V$. rosea and $A$. paniculuta in reducing seed borne and seed associated mycoflora and also increased the germination rate and vigor index.
\end{abstract}

Key words: Plant extracts, Seed germination, Seed associated fungi, Vigor index, Wheat seeds

\section{Introduction}

The cereal grains wheat (Triticum aestivum L.) is an important food crop all over the world. Planting cereal seed that is free of seed-borne pathogens is the primary means of limiting the introduction of pathogens, especially new pathogens, into a field. Planting infected seed may also result in widespread distribution of disease within the crop, and allows for an increased number of initial infection sites from which the disease can spread (Khair et al. 2003).

In Bangladesh 120 diseases are known to occur on wheat by different organisms and eight of them are caused by seed-borne fungi (Talukdar 1974, Fakir et al. 1977). Fungi cause the greatest impact with regard to diseases and crop production losses. Seed borne diseases of wheat like kernel bunt (Tilletia indica), loose smut (Ustilago nuda var tritici), head blight or scab (Fusarium spp.) and tundu or ear cockle (Clavibacter tritici and Anguina triticı) are considered as the constraints in wheat cultivation that affect crop yield and grain quality (Kumar et al. 2008). The storage fungi cause discoloration of seeds, deterioration of quality and decrease in germination. In addition, diseased seed may have toxic effects on humans and animals.

Large numbers of fungicides are being used in the form of dusting, slurry and soaking treatment. It is now realized that chemical fungicides cause serious environmental problems and are toxic to non-target organisms (Anon 2005). Extracts of many higher plants have been reported to exhibit antifungal properties under laboratory trials (Parekh et al. 2006, Mohana et al. 2008, Hajihassami et al. 2012, Yassin et al. 2012). Exploitation of plant metabolites in crop protection and prevention of bio deterioration caused by fungi appear to be promising. In view of these, the study was conducted to screen plant extracts against the germination, vigor index of seeds and seed associated fungi of wheat.

\footnotetext{
*Author for correspondence: mini_mahal@ru.ac.bd
} 


\section{Materials and Methods}

This experiment was conducted at the Plant Pathology Laboratory of the Department of Botany, University of Rajshahi.

\section{Collection of seeds and health testing}

Seed sample of wheat varieties such as Sourav and Prodip were collected from Regional Wheat Research Center, Shaympur, Rajshahi (RWRC) during May 2013 to April 2014. Seed health test was done by standard blotter method (ISTA 1996). In this method, four hundred seeds were randomly taken from each sample. Blotting papers were soaked in sterile water and three layers of these were placed at the bottom of each 9 $\mathrm{cm}$ petri dish. In each petri dish, 25 seeds of Sourav and Prodip varieties were placed at equal distance. The petri dishes were then incubated at temperature $28 \pm 2^{\circ} \mathrm{C}$ under defused light for seven days. Treated and untreated control seeds were evaluated for determination of seed germination and vigor index using following formula:

Germination $(\%)=$ Number of seeds germinated/Total number of seeds used $\times 100$

Vigor index $=($ Mean of root length + Mean of shoot length) $\times$ Percentage of seed germination (Abdul-Baki and Andersen 1972).

After seven days of incubation, the occurrence of fungal infection on seeds, number of seed germination and growth of shoot and root were recorded very carefully from the petri dish. The occurrence of fungi was the noted with the help of stereo binocular microscope and compound microscope. The fungi from the seeds were identified by observing their growth characteristics of the incubated seeds in the blotter under stereoscopic microscope and also by preparation of slides, following the standard manual keys and technique outlined by different authors (Booth 1971, Subramanian 1971) and slides were examined under microscope whenever necessary.

\section{Collection of plant materials}

Five different plant species such as Adhatida vasica, Tagetes patula, Vinca rosea, Lowsonia alba and Andrographis paniculuta were collected from Rajshahi University campus. Fresh and healthy leaves of each plant samples were washed thoroughly with running tap water to remove any dust. Washed leaves were dried in air.

\section{Preparation of plant extracts}

The collected fresh clean plant parts were chopped and the extracts were prepared by following procedure (Ahmed et al. 2013). In this method, the chopped leaf samples weighted $50 \mathrm{gm}$ each of plants and grounded in a mortar and pestle by adding sterile distilled water at 1:1, 1:2 and 1:3 w/v ratios $(1: 1=50 \mathrm{gm}$ plant material crushed in $50 \mathrm{ml}$ distilled water). The extracts were filtered through cheese cloth. These were centrifuged at $3000 \mathrm{rpm}$ for $20 \mathrm{~min}$ and stored in a refrigerator at $4^{\circ} \mathrm{C}$ until used. Seeds were treated with the prepared plant extracts separately by dipping method for 30 minutes.

\section{Seed treatment with plant extracts}

Selected seed samples of wheat were treated following dipping and standard blotter method (ISTA 1996). The seeds were dipping in 1:1, 1:2 and 1:3 dilutions for 30 minutes in previously prepared Adhatoda vasica, Tagetes, Vinca rosea, Lowsonia alba and Andrographis paniculuta leaf extracts. After 30 minutes the treated seeds were soaked on blotting paper and placed on moist blotter to determine seed germination, vigor index and seed mycoflora. For control, the seeds were soaked in distilled water. Surface sterilized 
seeds with $1 \%$ sodium hypochloride $(\mathrm{NaOCl})$ solution for 2 minutes were also treated with plant extracts. Twenty-five seeds of each wheat variety were used for each replication in blotter test.

\section{Statistical analysis}

Experiments were conducted in triplicate consistency of results and statistical purpose. The data were expressed as mean and standard error (M $\pm S E$ ) using Microsoft Excel software 2013. $P<0.05$ was considered statistically significant in ANOVA test.

\section{Results and Discussion}

\section{Effect of plant extracts on seed germination and vigor index of selected varieties of wheat}

Five different plant extracts were used for the efficacy of seed germination, vigor index and status of seed borne and seed associated fungi. In case of Sourav, it was found that percent seed germination, shoot length and root length were significantly $(p \leq 0.05)$ varied with the treatments of different dilutions of plant extracts as compare to control (Table 1). The maximum germination percentage (98.67\%) was recorded at 1:1 dilution of gada extract and it increased germination by $37.84 \%$ over control and the lowest was $90.67 \%$ at $1: 3$ dilution of nayantara extract. The highest vigor index (1687.80) was observed at 1:2 dilution of gada and the lowest was 1241.60 at 1:1 dilution of kalomegh extract. Unsterilized Sourav variety showed the maximum germination percentage $(97.33 \%)$ at $1: 1$ dilution of mehedi and basok extracts and it increased germination by $36.99 \%$ over control and the lowest was $73.33 \%$ at $1: 3$ dilution of basok extract. The highest vigor index (1333.42) was recorded at 1:1 dilution of mehedi, and the lowest was 989.38 at 1:3 dilution of gada. Ahmed et al. (2013) reported that out of five different plants extracts viz. Allium sativum (garlic), Allamanda cathartica (Allamanda), Azadirechta indica (Neem), Swertia (Chirata) and Polygonum hydropiper (Bishkatali) with two dilutions (1:1 \& 1:2) garlic extract at 1:1 dilution gave best results for three varieties which successfully increased seed germination. This result is completely agreed with the present observations.

In Prodip, the maximum germination percentage (94.67\%) was found at 1:1 dilution of gada extract and it increased germination by $36.62 \%$ and the minimum was $69.33 \%$ at $1: 3$ dilution of mehedi (Table 2). The highest vigor index (1469.85) was recorded at 1:3 dilution of kalomegh and the lowest was 999.60 at 1:1 dilution basok extract. Without surface sterilized seed of Prodip variety showed the maximum germination percentage (97.33\%) at 1:1 dilution of basok and it increased germination by $38.35 \%$ over control and the lowest was $72 \%$ at 1:3 dilution of mehedi extract. The highest vigor index (1466.44) was recorded at 1:1 dilution of mehedi and the lowest was 958.27 at 1:3 dilution of basok. Hasan et al., 2005 observed that root length, shoot length and vigor index was significantly increased after application with alcoholic and water extracts of rhizomes of ginger, bulb of garlic and leaf of neem on seeds of wheat while the lowest result was recorded in untreated control. These findings are partial agreement with the present observations.

\section{Effect of Plant extracts on seed borne and seed associated mycoflora of selected varieties of wheat}

Five different plant extracts showed different level of anti-fungal activity in controlling the seed associated fungi of the tested wheat varieties (Sourav and Prodip). All the plant extracts have inhibitory effect and reduced the incidence of seed borne and seed associated fungi. 
Seed associated mycoflora of unsterilized and surface sterilized seeds with $1 \% \mathrm{NaOCl}$ of Sourav and Prodip showed significant $(p \leq 0.05)$ variation with the treatments of different dilutions of plant extracts (Table 1 and 2). In blotter test recovered fungi were identified as Penicillium spp., Aspergillus spp., Rhizopus sp., Trichoderma viride, Fusarium sp. and Bipolaris sorokiniana. In case of surface sterilized seeds, Aspergillus spp. and $B$. sorokiniana were the most dominant fungi observed in treated wheat varieties (Table 1 and 2) which were also reported in the previous study by Islam et al. (2015). At 1:1 dilution of all tested plant extracts was the most effective in controlling of all the seed associated fungi when the seeds were surface sterilized with $1 \% \mathrm{NaOCl}$. Seed mycoflora was decreasing with decreasing the dilutions of plant extracts. Baka (2014) tested 14 Egyptian wheat cultivars for seed-borne fungi. He isolated five seed-borne fungi viz., Aspergillus flavus, A. niger, Curvularia lunata, Fusarium moniliforme and Penicillium chrysogenum from the wheat cultivars. He showed that, aqueous extract of different plants reduces the fungal disease incidence. This result is partial agreement with the present observations.

In case of surface sterilized and unsterilized seeds of Sourav, at 1:1 dilution of all tested plant extracts totally controlled all isolated seed mycoflora. Fusarium spp. and $T$. viride were successfully controlled at all dilutions of Mehedi, Kalomegh and Basok extracts and Rhizopus sp. was totally controlled at all dilutions of Basok extracts in both cases. Aspergillus spp. was also successfully controlled at all dilutions of Nayantara and Gada extracts. Ahmed et al (2013) reported that out of five different plants extracts viz. Allium sativum (garlic), Allamanda cathartica (Allamanda), Azadirechta indica (Neem), Swertia (Chirata) and Bishkatali with two dilutions (1:1 \& 1:2) Garlic extract at 1:1 dilution gave best results for three varieties which successfully reduced seed-borne infection. This result is partial agree with the present observations.

Results presented in Table 2 for without sterilized and surface sterilized seeds of Prodip, it was indicated that all dilutions of Mehedi, Kalomegh and Basok extracts successfully controlled T. viride and Fusarium sp. in both cases and beside this, Aspergillus spp. was remarkably controlled with Nayantara and Gada extracts and Fusarium sp. was totally controlled with Gada extract in surface sterilized seeds. Chowdhury et al. (2005) found that the seed borne fungi of lentil viz. Fusarium sp. Curvularia sp., Penicillium sp., Aspergillus sp. and Alternaria sp. were reduced after treatment the lentil seed with alcoholic and water extracts of Zingiber offcinale, Allium sativum, A. cepa, Adhatoda vesica, Lawsonia alba, Azadirachta indica, Achyranthes aspera, Cuscuta reflexa, Vinca rosea and Nigilla sativa. This result is completely agreed with the present observations.

From these experiments specific plant extract could not show the efficacy of complete elimination of seed associated fungi from wheat seeds but it did not fail to show different levels antifungal effects. Through such effect varied from one host pathogen (fungus) to the others. 
Table 1. Effects of plant extracts on seed germination, shoot length, root length, vigor index and seed associated fungi in Sourav

\begin{tabular}{|c|c|c|c|c|c|c|c|c|c|c|c|c|c|}
\hline \multirow[b]{2}{*}{ Seed } & \multirow[b]{2}{*}{ Treatment } & \multirow[b]{2}{*}{ Dilution } & \multirow{2}{*}{$\begin{array}{c}\text { Germination } \\
(\%) \\
\text { Mean+SE }\end{array}$} & \multirow{2}{*}{$\begin{array}{c}\text { Increased } \\
\text { germination } \\
\text { (\%) over } \\
\text { control }\end{array}$} & \multirow{2}{*}{$\begin{array}{l}\text { Shoot length } \\
\quad(\mathrm{cm}) \\
\text { Mean } \pm \text { SE }\end{array}$} & \multirow{2}{*}{$\begin{array}{l}\text { Root length } \\
(\mathrm{cm}) \\
\text { Mean } \pm \text { SE }\end{array}$} & \multirow[b]{2}{*}{$\begin{array}{l}\text { Vigor } \\
\text { index }\end{array}$} & \multicolumn{6}{|c|}{ Seed borne infection* $(\%)$} \\
\hline & & & & & & & & $\begin{array}{l}\text { Penicillum } \\
\text { spp. }\end{array}$ & $\begin{array}{c}\text { Aspergillus } \\
\text { spp. }\end{array}$ & $\begin{array}{c}\text { Rhizopus } \\
\text { sp. }\end{array}$ & $\begin{array}{l}\text { Fusarium } \\
\text { sp. }\end{array}$ & $\begin{array}{c}\text { Bipolaris } \\
\text { sorokiniana }\end{array}$ & $\begin{array}{c}\text { Trichoderma } \\
\text { viride }\end{array}$ \\
\hline \multirow{15}{*}{$\begin{array}{l}\text { Surface } \\
\text { sterilized }\end{array}$} & \multirow{3}{*}{ Mehedi } & 1:1 & $96.00 \pm 2.31$ & 36.11 & $8.00 \pm 0.12$ & $6.40 \pm 0.12$ & 1382.40 & 0 & 0 & 0 & 0 & 0 & 0 \\
\hline & & $1: 2$ & $94.67 \pm 1.33$ & 35.22 & $8.17 \pm 0.09$ & $6.70 \pm 0.12$ & 1407.43 & 0 & 4 & 4 & 0 & 4 & 0 \\
\hline & & $1: 3$ & $93.67 \pm 3.53$ & 34.53 & $9.03 \pm 0.09$ & $6.87 \pm 0.19$ & 1489.35 & 4 & 8 & 8 & 0 & 8 & 0 \\
\hline & \multirow{3}{*}{ Nayantara } & $1: 1$ & $96.00 \pm 2.31$ & 36.11 & $7.73 \pm 0.15$ & $5.97 \pm 0.12$ & 1315.20 & 0 & 0 & 4 & 0 & 0 & 0 \\
\hline & & $1: 2$ & $93.33 \pm 1.33$ & 34.29 & $8.40 \pm 0.06$ & $6.40 \pm 0.06$ & 1381.28 & 8 & 0 & 8 & 4 & 4 & 8 \\
\hline & & $1: 3$ & $91.00 \pm 2.31$ & 32.60 & $9.00 \pm 0.06$ & $6.87 \pm 0.07$ & 1443.87 & 12 & 0 & 12 & 8 & 8 & 12 \\
\hline & \multirow{3}{*}{ Kalomegh } & $1: 1$ & $96.00 \pm 2.31$ & 36.11 & $7.27 \pm 0.07$ & $5.67 \pm 0.12$ & 1241.60 & 0 & 4 & 0 & 0 & 0 & 0 \\
\hline & & $1: 2$ & $93.33 \pm 2.67$ & 34.29 & $7.63 \pm 0.09$ & $6.67 \pm 0.12$ & 1334.62 & 8 & 8 & 0 & 0 & 8 & 0 \\
\hline & & $1: 3$ & $90.67 \pm 5.33$ & 32.36 & $8.13 \pm 0.09$ & $7.03 \pm 0.09$ & 1375.16 & 8 & 16 & 0 & 0 & 12 & 0 \\
\hline & \multirow{3}{*}{ Basok } & 1:1 & $97.00 \pm 0.58$ & 36.77 & $7.53 \pm 0.32$ & $6.27 \pm 0.12$ & 1338.60 & 0 & 0 & 0 & 0 & 0 & 0 \\
\hline & & $1: 2$ & $96.00 \pm 2.31$ & 36.11 & $7.73 \pm 0.09$ & $6.40 \pm 0.21$ & 1356.80 & 0 & 8 & 0 & 0 & 8 & 0 \\
\hline & & $1: 3$ & $93.33 \pm 1.33$ & 34.29 & $8.27 \pm 0.15$ & $6.77 \pm 0.09$ & 1403.06 & 4 & 12 & 0 & 0 & 16 & 0 \\
\hline & \multirow{3}{*}{ Gada } & 1:1 & $98.67 \pm 1.33$ & 37.84 & $8.57 \pm 0.18$ & $6.60 \pm 0.15$ & 1496.50 & 0 & 0 & 0 & 0 & 0 & 0 \\
\hline & & $1: 2$ & $97.00 \pm 0.58$ & 36.77 & $8.43 \pm 0.18$ & $8.97 \pm 0.24$ & 1687.80 & 8 & 0 & 8 & 0 & 4 & 0 \\
\hline & & $1: 3$ & $96.00 \pm 1.15$ & 36.11 & $8.40 \pm 0.21$ & $8.93 \pm 0.27$ & 1664.00 & 12 & 0 & 12 & 0 & 8 & 4 \\
\hline \multicolumn{2}{|c|}{ F value (LSD $p \leq 0.05)$} & \multicolumn{2}{|r|}{$24.526(1.315)$} & & $12.673(0.414)$ & \multicolumn{2}{|l|}{$38.020(0.435)$} & \multicolumn{6}{|c|}{$3.56(3.44)$} \\
\hline \multirow{15}{*}{$\begin{array}{l}\text { Without } \\
\text { surface } \\
\text { sterilized }\end{array}$} & \multirow{3}{*}{ Mehedi } & $1: 1$ & $97.33 \pm 1.33$ & 36.99 & $7.23 \pm 0.15$ & $6.47 \pm 0.15$ & 1333.42 & 4 & 0 & 4 & 0 & 0 & 0 \\
\hline & & $1: 2$ & $90.67 \pm 1.33$ & 32.36 & $7.90 \pm 0.06$ & $5.67 \pm 0.12$ & 1230.09 & 8 & 8 & 8 & 0 & 8 & 0 \\
\hline & & $1: 3$ & $81.33 \pm 1.33$ & 24.59 & $8.03 \pm 0.19$ & $5.53 \pm 0.15$ & 1103.38 & 12 & 16 & 12 & 0 & 12 & 0 \\
\hline & \multirow{3}{*}{ Nayantara } & $1: 1$ & $94.67 \pm 1.33$ & 35.22 & $7.07 \pm 0.22$ & $5.83 \pm 0.18$ & 1221.24 & 8 & 0 & 8 & 4 & 0 & 0 \\
\hline & & $1: 2$ & $86.67 \pm 1.33$ & 29.24 & $7.60 \pm 0.15$ & $6.40 \pm 0.12$ & 1213.38 & 12 & 0 & 12 & 4 & 12 & 12 \\
\hline & & $1: 3$ & $76.00 \pm 2.31$ & 19.30 & $8.27 \pm 0.15$ & $7.20 \pm 0.12$ & 1175.47 & 16 & 4 & 16 & 12 & 12 & 16 \\
\hline & \multirow{3}{*}{ Kalomegh } & $1: 1$ & $93.33 \pm 1.33$ & 34.29 & $7.60 \pm 0.17$ & $6.20 \pm 0.12$ & 1287.95 & 4 & 8 & 4 & 0 & 4 & 0 \\
\hline & & $1: 2$ & $84.00 \pm 2.31$ & 26.99 & $8.27 \pm 0.15$ & $6.87 \pm 0.19$ & 1271.20 & 12 & 8 & 12 & 0 & 12 & 0 \\
\hline & & $1: 3$ & $77.33 \pm 2.67$ & 20.69 & $8.30 \pm 0.12$ & $7.07 \pm 0.07$ & 1188.30 & 16 & 16 & 16 & 0 & 16 & 0 \\
\hline & \multirow{3}{*}{ Basok } & $1: 1$ & $97.33 \pm 1.33$ & 36.99 & $8.03 \pm 0.19$ & $5.53 \pm 0.15$ & 1320.44 & 0 & 4 & 0 & 0 & 4 & 0 \\
\hline & & $1: 2$ & $81.33 \pm 1.33$ & 24.59 & $7.73 \pm 0.09$ & $6.40 \pm 0.21$ & 1149.46 & 4 & 12 & 0 & 0 & 16 & 0 \\
\hline & & $1: 3$ & $73.33 \pm 2.67$ & 16.36 & $7.23 \pm 0.15$ & $6.47 \pm 0.15$ & 1004.62 & 8 & 16 & 0 & 0 & 20 & 0 \\
\hline & \multirow{3}{*}{ Gada } & $1: 1$ & $93.33 \pm 2.67$ & 34.29 & $7.23 \pm 0.15$ & $6.47 \pm 0.15$ & 1278.62 & 8 & 4 & 4 & 0 & 4 & 0 \\
\hline & & $1: 2$ & $77.33 \pm 1.33$ & 20.69 & $7.57 \pm 0.27$ & $7.40 \pm 0.15$ & 1157.37 & 12 & 12 & 12 & 4 & 8 & 0 \\
\hline & & $1: 3$ & $70.67 \pm 1.33$ & 13.22 & $7.60 \pm 0.15$ & $6.40 \pm 0.12$ & 989.38 & 16 & 20 & 16 & 12 & 12 & 8 \\
\hline Control & & & $61.33 \pm 1.33$ & & $4.57 \pm 0.07$ & $4.10 \pm 0.06$ & 531.73 & 44 & 52 & 43 & 28 & 42 & 24 \\
\hline F value & $\mathrm{D}_{\mathrm{p} \leq 0.05)}$ & & 13.772 (1.512) & & $6.373(0.470)$ & $16.300(0.415)$ & & & & & (3.04) & & \\
\hline
\end{tabular}

*Mean of three replications. 
Table 2. Effects of plant extracts on seed germination, shoot length, root length, vigor index and seed associated fungi in Prodip

\begin{tabular}{|c|c|c|c|c|c|c|c|c|c|c|c|c|c|}
\hline \multirow{2}{*}{ Seed } & \multirow{2}{*}{ Treatment } & \multirow{2}{*}{ Dilution } & \multirow{2}{*}{$\begin{array}{c}\text { Germination } \\
(\%) \\
\text { Mean+SE }\end{array}$} & \multirow{2}{*}{$\begin{array}{l}\text { Increased } \\
\text { germination } \\
\text { (\%) over } \\
\text { control }\end{array}$} & \multirow{2}{*}{$\begin{array}{l}\text { Shoot length } \\
(\mathrm{cm}) \\
\text { Mean } \pm \text { SE }\end{array}$} & \multirow{2}{*}{$\begin{array}{l}\text { Root length }(\mathrm{cm}) \\
\text { Mean } \pm \mathrm{SE}\end{array}$} & \multirow{2}{*}{$\begin{array}{l}\text { Vigor } \\
\text { index }\end{array}$} & \multicolumn{6}{|c|}{ Seed borne infection (\%)* } \\
\hline & & & & & & & & \begin{tabular}{|c|}
$\begin{array}{c}\text { Penicillum } \\
\text { spp. }\end{array}$ \\
\end{tabular} & $\begin{array}{c}\text { Aspergillus } \\
\text { spp. }\end{array}$ & $\begin{array}{l}\text { Rhizopus } \\
\text { sp. }\end{array}$ & \begin{tabular}{|l|}
$\begin{array}{c}\text { Fusarium } \\
\text { sp. }\end{array}$ \\
\end{tabular} & $\begin{array}{c}\text { Bipolaris } \\
\text { sorokiniana } \\
\end{array}$ & $\begin{array}{c}\begin{array}{c}\text { Trichoderma } \\
\text { viride }\end{array} \\
\end{array}$ \\
\hline \multirow{15}{*}{$\begin{array}{l}\text { Surface } \\
\text { sterilized }\end{array}$} & \multirow{3}{*}{ Mehedi } & $1: 1$ & $92.00 \pm 2.31$ & 34.78 & $8.43 \pm 0.12$ & $6.57 \pm 0.12$ & 1380.00 & 0 & 0 & 0 & 0 & 0 & 0 \\
\hline & & $1: 2$ & $84.00 \pm 2.31$ & 28.57 & $8.17 \pm 0.09$ & $6.70 \pm 0.12$ & 1248.80 & 0 & 4 & 4 & 0 & 4 & 0 \\
\hline & & $1: 3$ & $69.33 \pm 2.67$ & 13.46 & $8.40 \pm 0.21$ & $8.93 \pm 0.27$ & 1201.72 & 8 & 12 & 8 & 0 & 8 & 0 \\
\hline & \multirow{3}{*}{ Nayantara } & $1: 1$ & $90.67 \pm 1.33$ & 33.83 & $7.73 \pm 0.15$ & $5.97 \pm 0.12$ & 1242.18 & 4 & 0 & 4 & 0 & 0 & 0 \\
\hline & & $1: 2$ & $86.67 \pm 1.33$ & 30.77 & $7.63 \pm 0.09$ & $6.67 \pm 0.12$ & 1239.38 & 8 & 0 & 8 & 4 & 4 & 4 \\
\hline & & $1: 3$ & $77.33 \pm 2.67$ & 22.41 & $8.27 \pm 0.15$ & $6.77 \pm 0.09$ & 1162.53 & 16 & 0 & 16 & 8 & 8 & 8 \\
\hline & \multirow{3}{*}{ Kalomegh } & $1: 1$ & $93.33 \pm 1.33$ & 35.71 & $7.23 \pm 0.15$ & $6.47 \pm 0.15$ & 1278.62 & 0 & 4 & 0 & 0 & 0 & 0 \\
\hline & & $1: 2$ & $78.67 \pm 1.33$ & 23.73 & $7.63 \pm 0.09$ & $6.67 \pm 0.12$ & 1124.98 & 8 & 8 & 0 & 0 & 4 & 0 \\
\hline & & $1: 3$ & $70.67 \pm 2.67$ & 15.10 & $13.77 \pm 5.62$ & $7.40 \pm 0.55$ & 1495.85 & 8 & 16 & 0 & 0 & 12 & 0 \\
\hline & \multirow{3}{*}{ Basok } & $1: 1$ & $84.00 \pm 2.31$ & 28.57 & $6.83 \pm 0.18$ & $5.07 \pm 0.30$ & 999.60 & 0 & 4 & 0 & 0 & 4 & 0 \\
\hline & & $1: 2$ & $84.00 \pm 2.31$ & 28.57 & $8.17 \pm 0.09$ & $6.70 \pm 0.12$ & 1248.80 & 0 & 8 & 0 & 0 & 8 & 0 \\
\hline & & $1: 3$ & $76.00 \pm 2.31$ & 21.05 & $8.27 \pm 0.15$ & $7.20 \pm 0.12$ & 1175.47 & 4 & 20 & 0 & 0 & 16 & 0 \\
\hline & \multirow{3}{*}{ Gada } & $1: 1$ & $94.67 \pm 1.33$ & 36.62 & $7.23 \pm 0.15$ & $6.47 \pm 0.15$ & 1296.98 & 4 & 0 & 0 & 0 & 0 & 0 \\
\hline & & $1: 2$ & $82.67 \pm 3.53$ & 27.42 & $7.53 \pm 0.20$ & $8.00 \pm 0.26$ & 1284.14 & 8 & 0 & 8 & 0 & 4 & 0 \\
\hline & & $1: 3$ & $72.00 \pm 2.31$ & 16.67 & $8.13 \pm 0.20$ & $8.83 \pm 0.18$ & 1221.60 & 12 & 0 & 12 & 0 & 8 & 4 \\
\hline \multicolumn{2}{|c|}{ F value (LSD $p \leq 0.05$ ) } & \multicolumn{2}{|c|}{13.684 (1.611) } & \multicolumn{2}{|c|}{$1.215(4.209)$} & $21.436(0.630)$ & \multicolumn{7}{|c|}{$7.00(2.81)$} \\
\hline \multirow{15}{*}{$\begin{array}{l}\text { Without } \\
\text { Surface } \\
\text { sterilized }\end{array}$} & \multirow{3}{*}{ Mehedi } & 1:1 & $97.33 \pm 1.33$ & 38.35 & $8.50 \pm 0.17$ & $6.57 \pm 0.12$ & 1466.44 & 4 & 0 & 4 & 0 & 0 & 0 \\
\hline & & $1: 2$ & $90.67 \pm 1.33$ & 33.83 & $8.27 \pm 0.15$ & $6.87 \pm 0.19$ & 1372.14 & 8 & 8 & 8 & 0 & 8 & 0 \\
\hline & & $1: 3$ & $72.00 \pm 2.31$ & 16.67 & $8.30 \pm 0.12$ & $7.07 \pm 0.07$ & 1106.40 & 12 & 16 & 12 & 0 & 12 & 0 \\
\hline & \multirow{3}{*}{ Nayantara } & $1: 1$ & $92.00 \pm 2.31$ & 34.78 & $7.07 \pm 0.22$ & $5.83 \pm 0.18$ & 1186.80 & 8 & 0 & 8 & 4 & 0 & 0 \\
\hline & & $1: 2$ & $84.00 \pm 2.31$ & 28.57 & $8.27 \pm 0.15$ & $6.87 \pm 0.19$ & 1271.20 & 12 & 0 & 12 & 4 & 12 & 8 \\
\hline & & $1: 3$ & $77.33 \pm 2.67$ & 22.41 & $7.57 \pm 0.27$ & $7.40 \pm 0.15$ & 1157.37 & 20 & 4 & 16 & 12 & 12 & 12 \\
\hline & \multirow{3}{*}{ Kalomegh } & $1: 1$ & $93.33 \pm 1.33$ & 35.71 & $7.30 \pm 0.35$ & $6.73 \pm 0.12$ & 1309.73 & 4 & 8 & 4 & 0 & 4 & 0 \\
\hline & & $1: 2$ & $84.00 \pm 2.31$ & 28.57 & $8.50 \pm 0.17$ & $6.57 \pm 0.12$ & 1265.60 & 12 & 8 & 12 & 0 & 12 & 0 \\
\hline & & $1: 3$ & $76.00 \pm 2.31$ & 21.05 & $7.73 \pm 0.09$ & $6.40 \pm 0.21$ & 1074.13 & 20 & 16 & 16 & 0 & 16 & 0 \\
\hline & \multirow{3}{*}{ Basok } & $1: 1$ & $93.33 \pm 1.33$ & 35.71 & $8.27 \pm 0.15$ & $7.20 \pm 0.12$ & 1443.50 & 0 & 8 & 0 & 0 & 4 & 0 \\
\hline & & $1: 2$ & $84.00 \pm 2.31$ & 28.57 & $7.43 \pm 0.26$ & $6.40 \pm 0.12$ & 1162.00 & 4 & 16 & 0 & 0 & 16 & 0 \\
\hline & & $1: 3$ & $74.67 \pm 2.67$ & 19.65 & $7.07 \pm 0.22$ & $5.83 \pm 0.18$ & 963.24 & 8 & 24 & 0 & 0 & 20 & 0 \\
\hline & \multirow{3}{*}{ Gada } & $1: 1$ & $92.00 \pm 2.31$ & 34.78 & $8.50 \pm 0.17$ & $6.57 \pm 0.12$ & 1386.13 & 8 & 4 & 4 & 0 & 4 & 0 \\
\hline & & $1: 2$ & $84.00 \pm 2.31$ & 28.57 & $7.07 \pm 0.19$ & $7.20 \pm 0.51$ & 1198.40 & 12 & 12 & 12 & 4 & 8 & 0 \\
\hline & & $1: 3$ & $74.67 \pm 3.53$ & 19.65 & $8.30 \pm 0.12$ & $7.07 \pm 0.07$ & 1147.43 & 16 & 20 & 16 & 12 & 12 & 8 \\
\hline Control & & & $60.00 \pm 2.31$ & & $4.03 \pm 0.12$ & $3.33 \pm 0.09$ & 441.60 & 39 & 49 & 33 & 32 & 40 & 25 \\
\hline F value ( & $D p \leq 0.05)$ & - & $13.249(1.630)$ & - & $8.487(0.568)$ & $5.896(0.555)$ & - & & & & $2.85)$ & & \\
\hline
\end{tabular}

*Mean of three replications. 

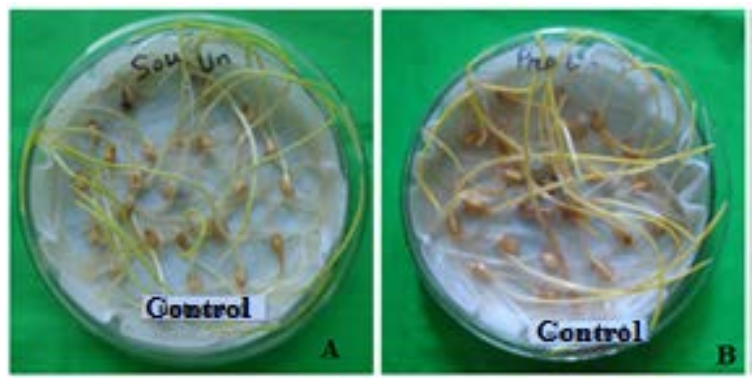

Fig. 1. Photograph showing infected seed and germination rate of $(A=$ Sourav and $B=$ Prodip $)$ wheat varieties in control condition.
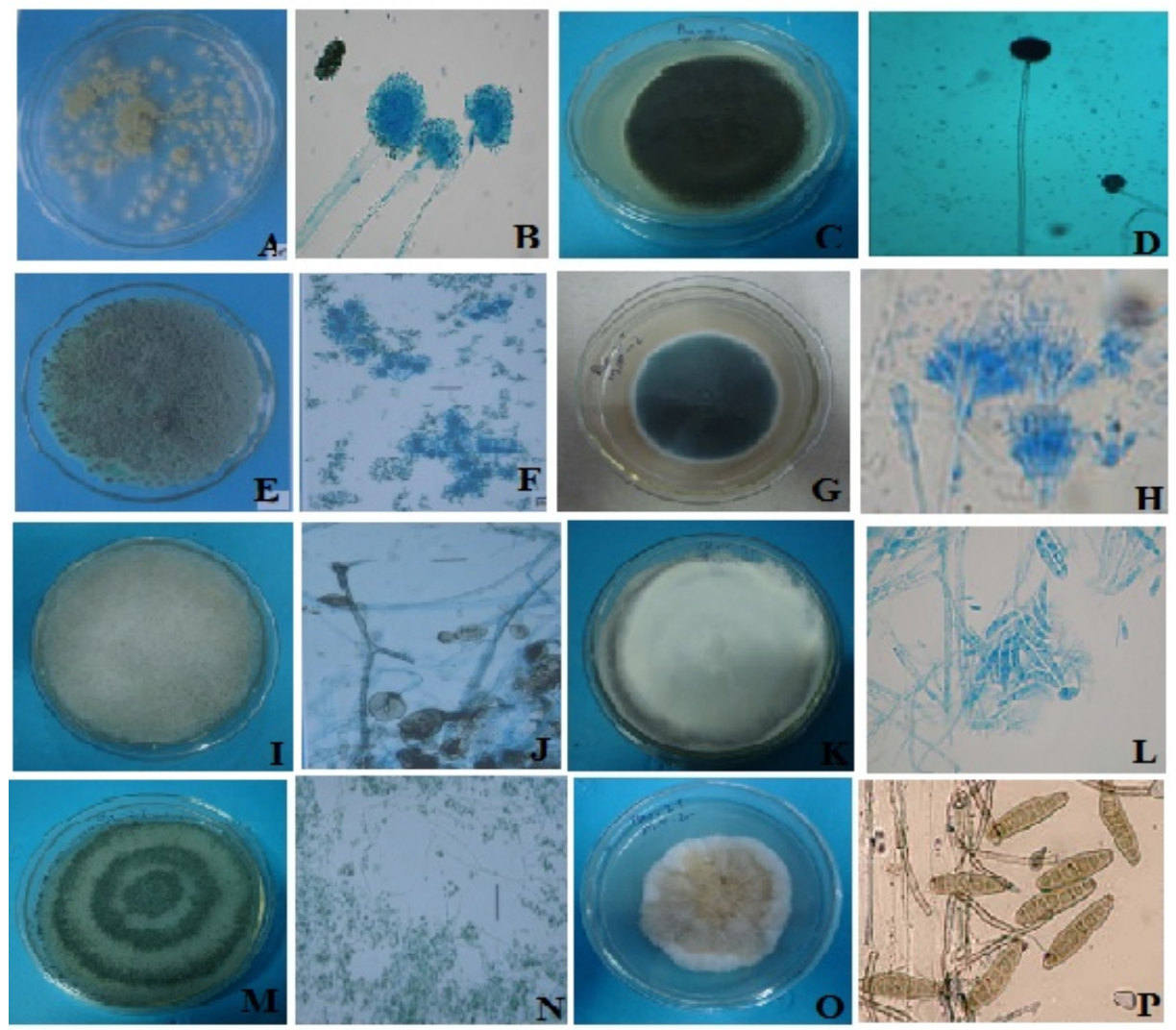

Fig. 2. Photograph showing different species of fungi. $A, C, E, G, I, K$ and $M=$ Culture plate, $B, D, F, H, J, L$ and $\mathrm{N}=$ Conidia and Conidiophores. Here,

$\mathrm{A}$ and $\mathrm{B}=$ Aspergillus flavus

$\mathrm{E}$ and $\mathrm{F}=$ Penicillium sp.

$\mathrm{I}$ and $\mathrm{J}=$ Rhizopus $\mathrm{sp}$.

$\mathrm{M}$ and $\mathrm{N}=$ Trichoderma viride
$\mathrm{C}$ and $\mathrm{D}=$ Aspergillus niger

$\mathrm{G}$ and $\mathrm{H}=$ Penicillium $\mathrm{sp}$.

$\mathrm{K}$ and $\mathrm{L}=$ Fusarium $\mathrm{sp}$.

$\mathrm{O}$ and $\mathrm{P}=$ Bipolaris sorokiniana 


\section{References}

Anon (2005). Pest control background. International Journal of Pest Control, 45 (2): 232-233.

Abdul-Baki AA and Anderson JD (1972). Physiological and Bio- chemical deterioration of seeds, In: Seed Biology, Vol. 2: TT Kozlowski (Ed.), Academic Press, New York, pp. 283-315.

Ahmed MM, Hossain K, Hassan C and Dash K (2013). Efficacy of different plant extracts on reducing seed-borne infection and increasing germination of collected rice seed sample. Universal Journal of plant Science, 1(3): 66-73.

Baka MZ (2014). Plant extract control of the fungi associated with different Egyptian wheat cultivars grains. Journal of Plant Protection Research, 54(3): 231-237.

Booth C (1971). The Genus Fusarium. Commonwealth Mycological Institute. Kew, Surrey, England.

Chowdhury SP, Hasan MM, Alam S, Chowdhury AN and Alam MS (2005). Effect of plant extracts on seed borne fungi of lentil (Lens esculenta Moench). Bangladesh Journal of Crop Science, 16(2): 197-205.

Fakir GA, Khan AL, Neergaard P and Mathur SB (1977). Transmission of Drechslera spp. through wheat seed in Bangladesh. Bangladesh Journal of Agricultural Research, 1: 113-118.

Hasan MM, Chowdhury SP, Alam S, Hossain B and Alam MS (2005). Antifungal effect of plant extracts on seed-borne fungi of wheat seeds regarding seed germination, seedling health and vigor index. Pakistan Journal Biological Sciences, 8(9): 1284-1289

Hajihasani M, Hajihassani A and Khaghani S (2012). Incidence and distribution of seed-borne fungi. Journal of Biotechnology, 11(23): 6290-6295

ISTA (1996). International rules for seed testing. International Seed Testing Association. Switzerland.

Islam MS, Sarker MNI and Ali MA (2015). Effect of seed borne fungi on germinating wheat seed and their treatment with chemicals. International Journal of Natural and Social Sciences, 2(1): 28-32.

Khair A, Alam N and Islam ABM (2003). Activity of some biological products against seed borne fungi of rice. Bangladesh Journal of Life Sciences, 15(2): 29-34.

Kumar A, Singh US, Kumar J and Garg GK (2008). Application of molecular and immune diagnostic tools for detection, surveillance and quarantine regulation of Karnal bunt (Tilletia indica) of wheat. Food and Agricultural Immunology, 19(4): 293-311.

Mohana DC, Raveesha KA and Lokanath R (2008). Herbal remedies for the management of seed-borne fungal pathogens by an edible plant Decalepis hamiltonii (Wight \& Arn). Archives of Phytopathology and Plant Protection, 41(1): 38-49.

Parekh J, Karathia N and Chanda S (2006). Evaluation of antibacterial activity and phytochemical analysis of Bauhinia variegata L. bark. African Journal of Biomedical Research, 9: 53-56.

Subramanian CV (1971). Hypomycetes. Indian Council of Agricultural Research, New Delhi.

Talukdar MJ (1974). Plant disease in Bangladesh. Bangladesh Journal of Agricultural Research, 1: 61-83.

Yassin MA, Moslem MA and El-Samawaty A (2012). Mycotoxins and non-fungicidal control of corn grain rotting fungi. Journal of Plant Science, 7(3): 96-104. 